\title{
A Low-Voltage Operational Transconductance Amplifier and Its Application to a Bandpass Gm-C Filter
}

\author{
C. Yoo and W. Kim
}

School of Electrical Engineering, Seoul National University

Room 301-817, Integrated Systems Lab., School of Electrical Engineering, Seoul National University, San 56-1, Shilim-Dong, Gwanak-Gu, Seoul 151-742, Korea

Phone: +82 2880 7466, Fax: +822882 4656, e-mail : \{changsik,wkim\}@iclab.snu.ac.kr

\begin{abstract}
A low-voltage operational transconductance amplifier(OTA) and a bandpass $\mathrm{Gm}-\mathrm{C}$ filter built with the OTA are described in this paper. The OTA has effectively only two MOS transistors in saturation region between $V_{D D}$ and $G N D$ - suitable for low voltage operation, and there are no internal nodes in the OTA - suitable for high frequency operation. With this OTA, a prototype bandpass $\mathrm{Gm}-\mathrm{C}$ filter was implemented in $0.8 \mu \mathrm{m}$ CMOS process. The center frequency can be controlled from $15.0 \mathrm{MHz}$ to $33.0 \mathrm{MHz}$. The filter consumes only $0.43 \mathrm{~mW}$ per pole when the center frequency is $15.0 \mathrm{MHz}$.
\end{abstract}

\section{Keywords}

OTA, MOS transistor, low-voltage operation, low-power consumption, highfrequency operation, $\mathrm{Gm}-\mathrm{C}$ filter 


\section{INTRODUCTION}

Gm-C filters have been widely used for high frequency application because its open loop architecture provides high frequency capability (Stefanelli, 1993), (Khoury, 1991). However, as the supply voltage is scaled down for low-power consumption, high-frequency operation becomes difficult to be achieved due to the rapid performance degradation of OTA's.

MOS transistors in the linear operation region can be used in OTA's for lowvoltage operation, but it generally requires bipolar transistors to keep the drain-tosource voltage of the MOS transistors constant (Rezzi, 1995). As an alternative, B. Nauta used CMOS inverter as OTA and achieved $22.0 \mathrm{MHz}$ cut-off frequency with $2.5 \mathrm{~V}$ single power supply. This is due to the fact that CMOS inverters do not have internal nodes limiting the operation frequency. However, the supply voltage should be controlled to tune the frequency characteristics of the filter (Nauta, 1992).

In this paper, a differential low-voltage OTA is described which is designed to be simple for low power and high frequency operation. The common mode feedback is achieved with no additional power consumption, aiding low power operation. With the OTA, a prototype bandpass $\mathrm{Gm}-\mathrm{C}$ filter is implemented in a $0.8 \mu \mathrm{m}$ CMOS process whose center frequency can be controlled from $15.0 \mathrm{MHz}$ to $33.0 \mathrm{MHz}$ with $3.0 \mathrm{~V}$ single power supply.

\section{LOW-VOLTAGE OPERATIONAL TRANSCONDUCTANCE AMPLIFIER}

In Figure 1, the low-voltage OTA is shown with its bias circuit. The transistors $M_{1} \sim M_{4}$ are in the saturation region, and the transistors $M_{5}$ and $M_{6}$ are in the linear operation region. The basic voltage-to-current conversion is done by the transistors $M_{1}$ and $M_{2}$, and the transistors $M_{3}$ and $M_{4}$ act as the load. The common mode feedback is performed by the transistors $M_{5}$ and $M_{6}$. This common mode feedback loop requires no additional current consumption, aiding low power operation. Except for the source node of the transistors $M_{3}$ and $M_{4}$, there are no internal nodes in the OTA. So, the filter built with this OTA can achieve high-frequency operation. And because there are only two transistors in saturation region between $V_{D D}$ and $G N D$, the OTA can operate at low power supply voltage.

The output current of the OTA is given as follows; 


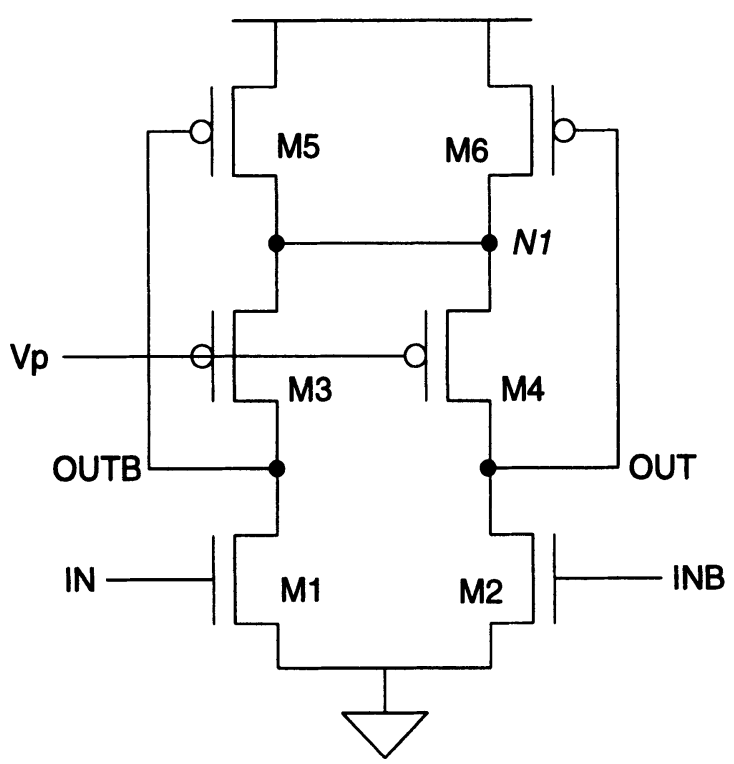

(a)

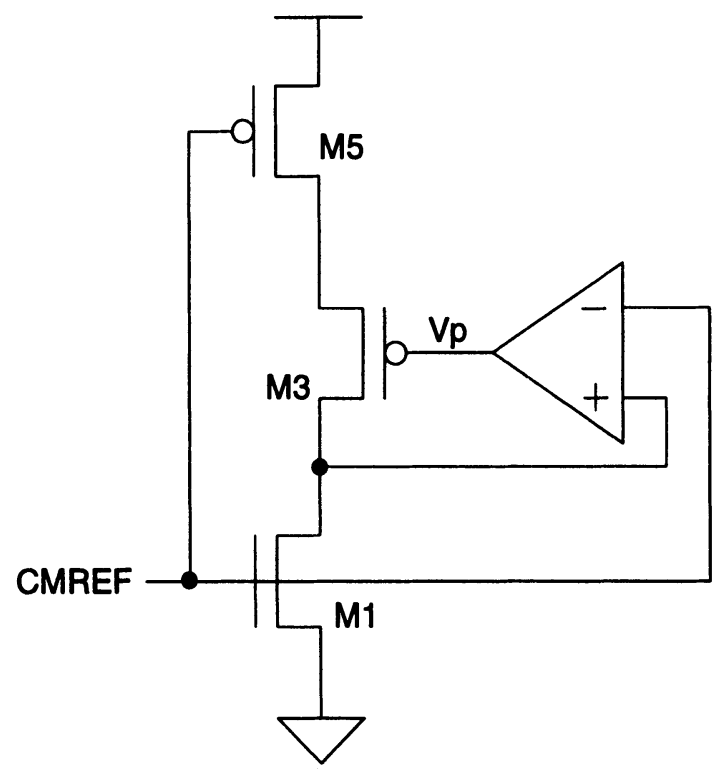

(b)

Figure 1 (a) Low-voltage, low-power operational transconductance amplifier. $M_{1}$, $M_{2}, M_{3}$ and $M_{4}$ are in the saturation region, and $M_{5}$ and $M_{6}$ which constitute common mode feedback loop are in the linear operation region. (b) Replica bias circuit for the OTA generating $V_{P}$ such that the output is CMREF when input is CMREF. 


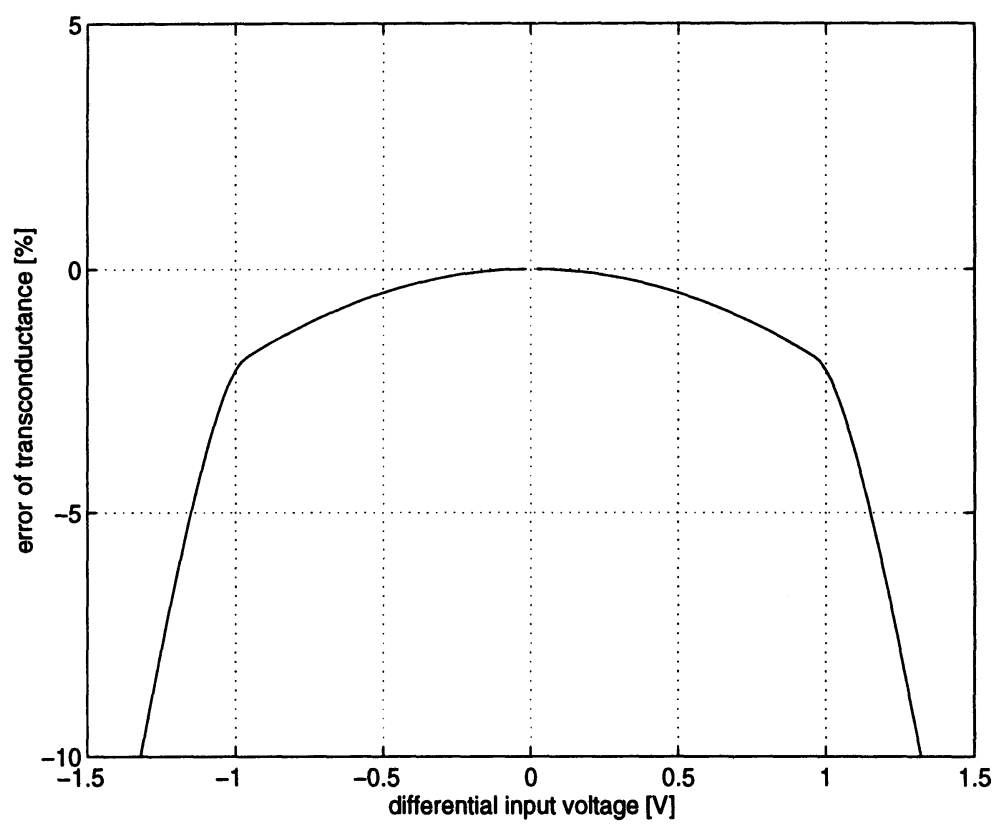

Figure 2 Linearity error as a function of differential input.

$$
i_{\text {out }}=\frac{1}{2} \beta_{1}\left(v_{G S 1}-v_{G S 2}\right)\left(v_{G S 1}+v_{G S 2}-2 V_{T}\right)
$$

where $\beta_{1}=\mu_{n} C_{O X}(W / L)_{1}$. From the equation (1), the transconductance of the OTA is;

$$
g_{m}=\frac{1}{2} \beta_{1}\left(v_{G S 1}+v_{G S 2}-2 V_{T}\right) .
$$

As can be seen in the equation (2), the transconductance of the OTA is determined by the size of the transistor $M_{1}$ and the common mode level of the input. Thus, in order to get a linearized transconductor, we must have stabilized common mode level of the input. Within a filter, this is achieved by the bias circuit shown in Figure 1-(b) and the common mode feedback loop consisting of the transistors $M_{5}$ and $M_{6}$, assuming that the input to the filter has stabilized common mode level. The replica bias circuit shown in Figure 1-(b) generates the bias voltage $V_{P}$ such that when the input voltage is $C M R E F$, the output voltage is also CMREF. The value of $C M R E F$ is $V_{D D} / 2$. 


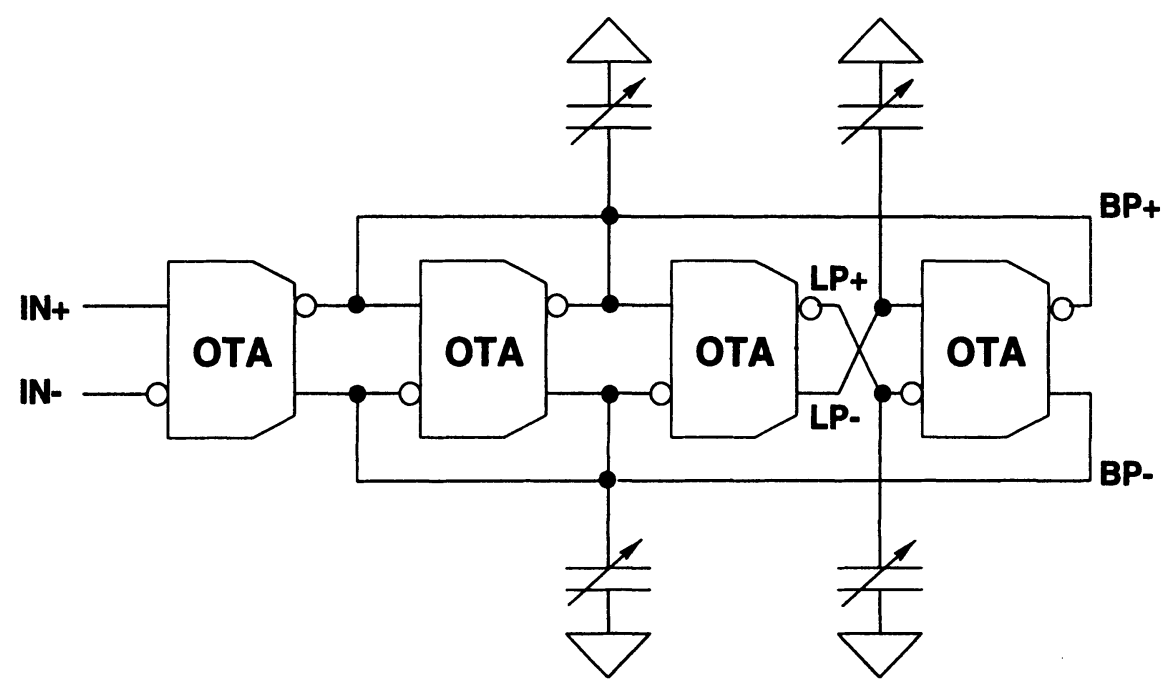

Figure 3 Second-order biquad circuit. The variable capacitors are to tune the frequency characteristic to the desired one when there are temperature variation and process drift. The variable capacitors are built with binary weighted capacitor array.

The size of the transistors of the replica bias circuit in Figure 1-(b) is same as that of the same numbered transistor of the OTA in Figure 1-(a).

Figure 2 shows the linearity error as a function of differential input. As can be seen, the error is smaller than $2.0 \%$ for $-1.0 \mathrm{~V}<v_{\text {in }}<1.0 \mathrm{~V}$ at DC. The linearity error is defined as;

$$
\text { error }=\frac{i_{\text {out }}-i_{\text {out }}(0)-g_{m}(0) v_{\text {in }}}{g_{m}(0) v_{\text {in }}} \times 100[\%]
$$

where $v_{i n}$ is the differential input, $i_{\text {out }}(0)$ and $g_{m}(0)$ are the output current and transconductance when $v_{\text {in }}=0 \mathrm{~V}$, respectively, and $i_{\text {out }}$ is the output current.

\section{SIXTH-ORDER BANDPASS GM-C FILTER}

In order to verify the usefulness of the low-voltage, low-power OTA described in previous section, a sixth-order bandpass $\mathrm{Gm}-\mathrm{C}$ filter is implemented by cascading three biquadratic sections shown in Figure 3. 


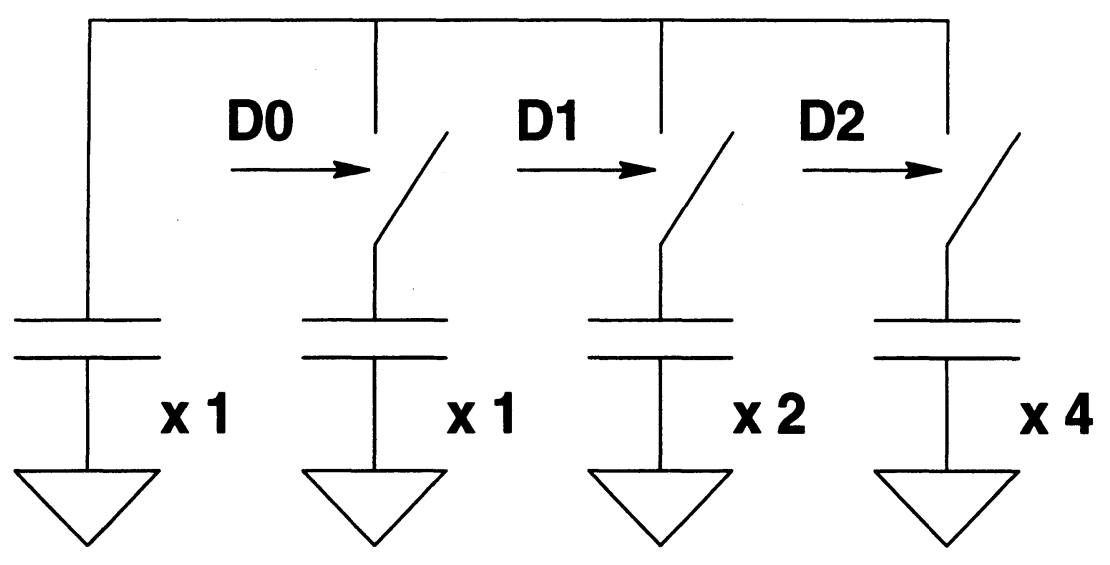

Figure 4 Binary weighted capacitor array. The capacitance is controlled with the digital control signal $\mathrm{D}[2: 0]$.

The center frequency of the filter is controlled by the variable load capacitors instead of the transconductance of the OTA. This is because the transconductance of the OTA cannot be changed if the size of the transistors and the common mode level of the input are determined once. The tunability of the frequency characteristic of $\mathrm{Gm}-\mathrm{C}$ filter is a must because the frequency characteristics of continuous-time filters can change as much as $\pm 50 \%$ due to process drift, temperature variation, aging, and etc (Yoo, 1996), (Gopinathan, 1990), (Khorramabadi, 1996).

The variable capacitors are built with the binary-weighted capacitor array shown in Figure 4. The capacitance is controlled by the digital signal $D[2: 0]$. The control range can be extended easily if desired.

Although the capacitance has to be controlled manually in the present implementation, it can also be controlled automatically by monitoring the frequency characteristic of the filter as in conventional master-slave tuning scheme (Yoo, 1996), (Gopinathan, 1990). There are several works related to this issue, and they can be applied to this filter without any difficulties (Khorramabadi, 1996).

\section{EXPERIMENTAL RESULTS}

A prototype sixth-order bandpass $\mathrm{Gm}-\mathrm{C}$ filter was implemented in $0.8 \mu \mathrm{m}$ doublemetal CMOS process, and occupies $1.0 \mathrm{~mm} \times 0.4 \mathrm{~mm}$ of silicon area. 
Table 1 Performance comparison with other CMOS continuous-time filters.

\begin{tabular}{|l|l|l|l|l|}
\hline Reference & $f_{\text {cut-off }}[\mathrm{kHz}]$ & $N$ & Power $[\mathrm{mW}]$ & $F M$ \\
\hline Khoury, 1991 & 15,000 & 5 & 96 & 2.89 \\
\hline Kaiser, 1989 & 0.945 & 3 & 0.0126 & 2.35 \\
\hline Huang, 1995 & 560 & 2 & 2.5 & 2.65 \\
\hline This work & 15,000 & 6 & 2.58 & 4.54 \\
\hline
\end{tabular}

The center frequency can be controlled from $15.0 \mathrm{MHz}$ to $33.0 \mathrm{MHz}$ by varying the size of capacitors with digital control signal $\mathrm{D}[2: 0]$. The filter consumes only $2.6 \mathrm{~mW}$ with $3.0 \mathrm{~V}$ single power supply. Peak-to-peak differential input can be applied upto $0.85 \mathrm{~V}$ for $1.0 \%$ total harmonic distortion when the center frequency is $15.0 \mathrm{MHz}$.

The frequency characteristic of the filter is shown in Figure 5. The passband gain appears to become smaller as the center frequency gets higher because the output buffer and test set-ups have lowpass characteristic. But the filter itself has constant passband gain regardless of its center frequency.

The transient response of the filter is measured and the spectrum of the filtered output is observed with the center frequency controlled to be $15.0 \mathrm{MHz}$.

When the input of $1.0 \mathrm{MHz}$ sinusoid(in stopband) added to $15.0 \mathrm{MHz}$ sinusoid(in passband) is applied, the filtered output is shown in the bottom trace of Figure 5(a) with the input in the top trace. Figure 6-(b) is the spectrum of the filtered output. Only the $15.0 \mathrm{MHz}$ component in the passband can be seen.

Figure 7-(a) and (b) are the waveforms of input and output and the spectrum of the output respectively with the input of $14.0 \mathrm{MHz}$ sinusoid added to $16.0 \mathrm{MHz}$ sinusoid(both of them are in the passband). Both of $14.0 \mathrm{MHz}$ and $16.0 \mathrm{MHz}$ components can be seen in the filtered output.

The power consumption of the filter is compared with those of ones presented elsewhere based on the figure of merit defined as;

$$
F M=\log \left(\frac{f_{\text {cut }- \text { off }}}{\text { Power } / N}\right)
$$

where $f_{\text {cut-off }}$ is the center or cut-off frequency of filter in $\mathrm{kHz}$ and Power $/ \mathrm{N}$ is the power per pole in $\mathrm{mW}$. The figure of merits of some works are summarized in Table 1. We can see that the prototype filter has the advantage of low power consumption. 


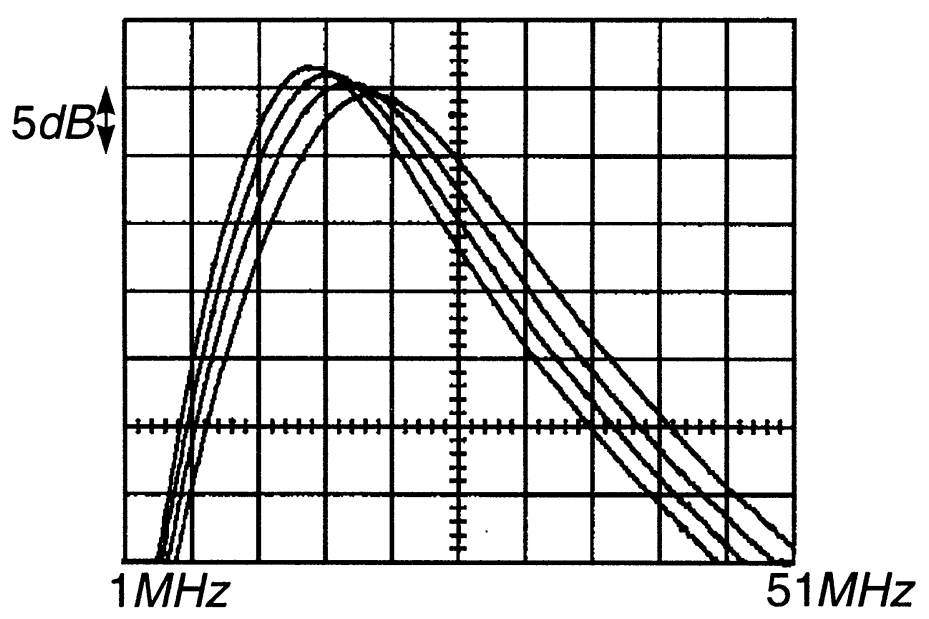

(a)

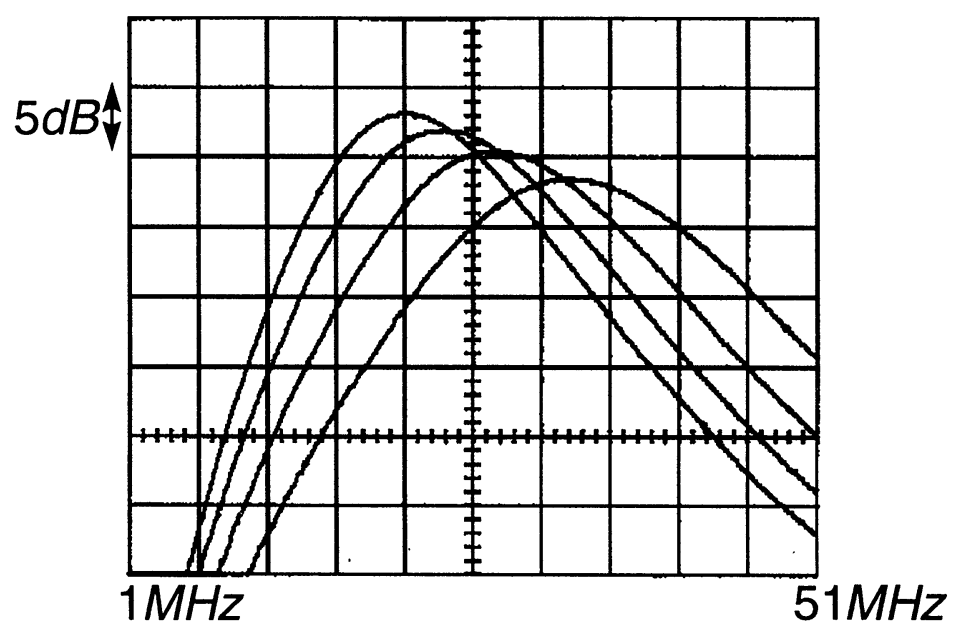

(b)

Figure 5 Frequency characteristic of sixth-order bandpass $\mathrm{Gm}-\mathrm{C}$ filter. (a) When $\mathrm{D}[2: 0]$ is $000 \sim 011$, (b) When $\mathrm{D}[2: 0]$ is 100 111. Center frequency can be controlled from $15.0 \mathrm{MHz}$ to $33.0 \mathrm{MHz}$. The passband gain gets smaller as the center frequency becomes larger due to the lowpass characteristic of test set-ups and output buffer. 


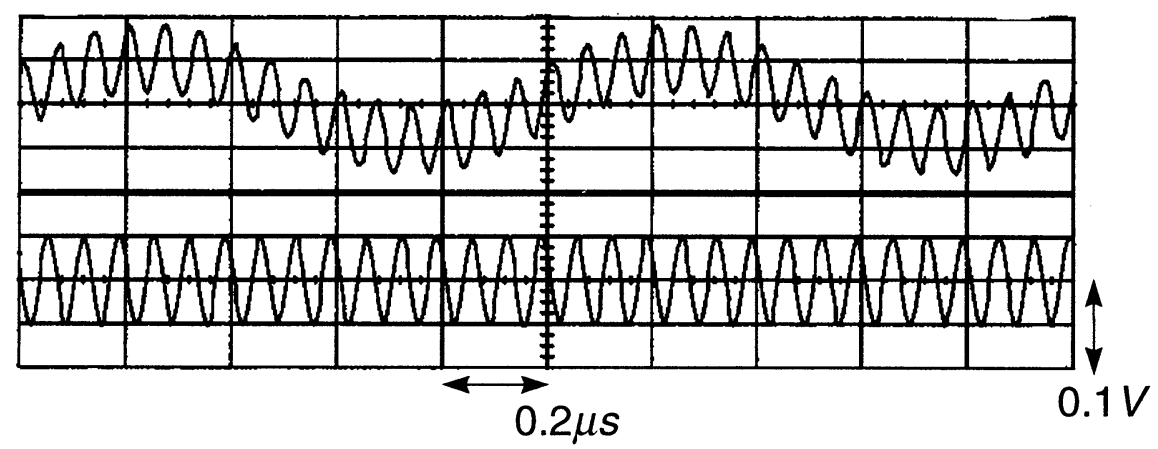

(a)

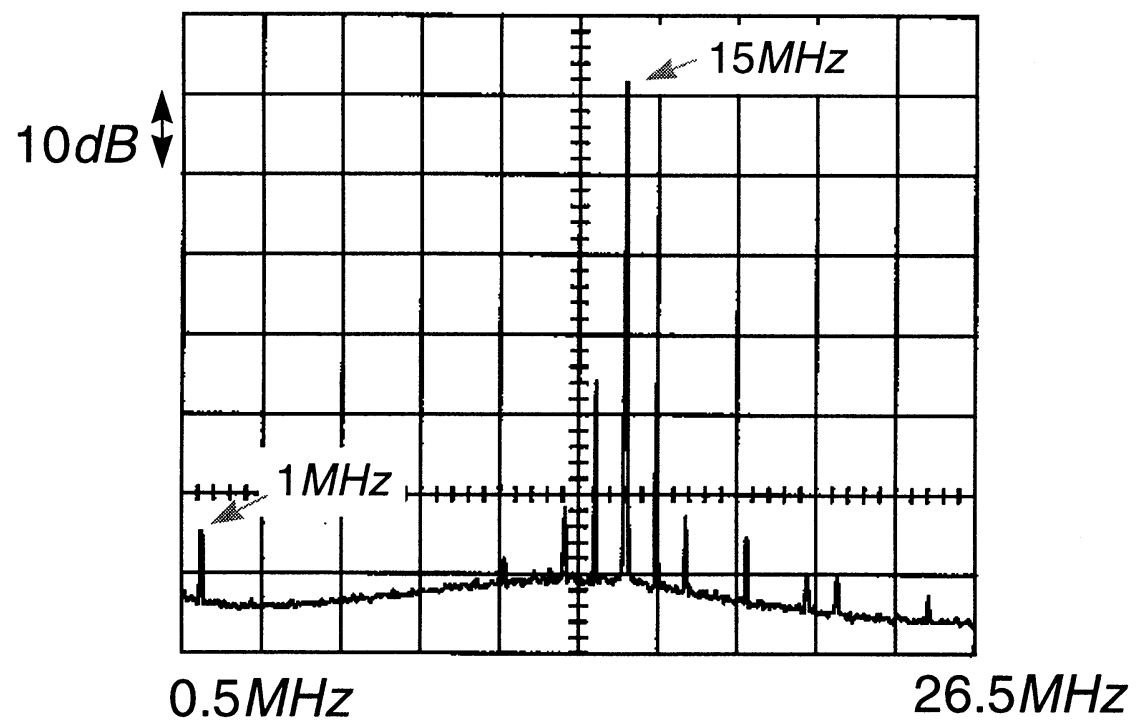

(b)

Figure 6 (a) Transient response when $D[2: 0]=000$, that is, cut-off frequency $=$ $15.0 \mathrm{MHz}$. Top trace $=$ input, sum of $1.0 \mathrm{MHz}$ and $15.0 \mathrm{MHz}$ sinusoid. Bottom trace = filtered output. (b) Spectrum of the output in (a). It can be seen $1.0 \mathrm{MHz}$ component is attenuated. 


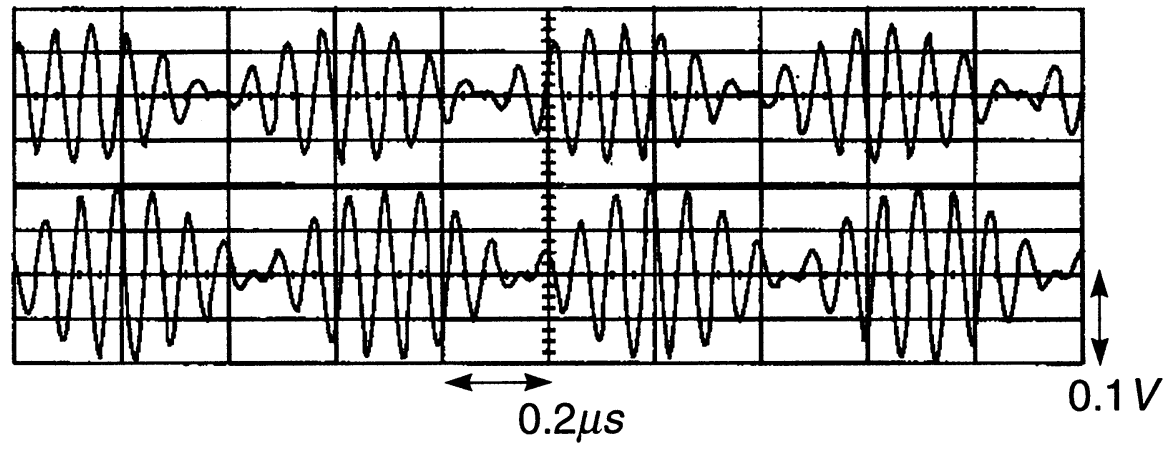

(a)

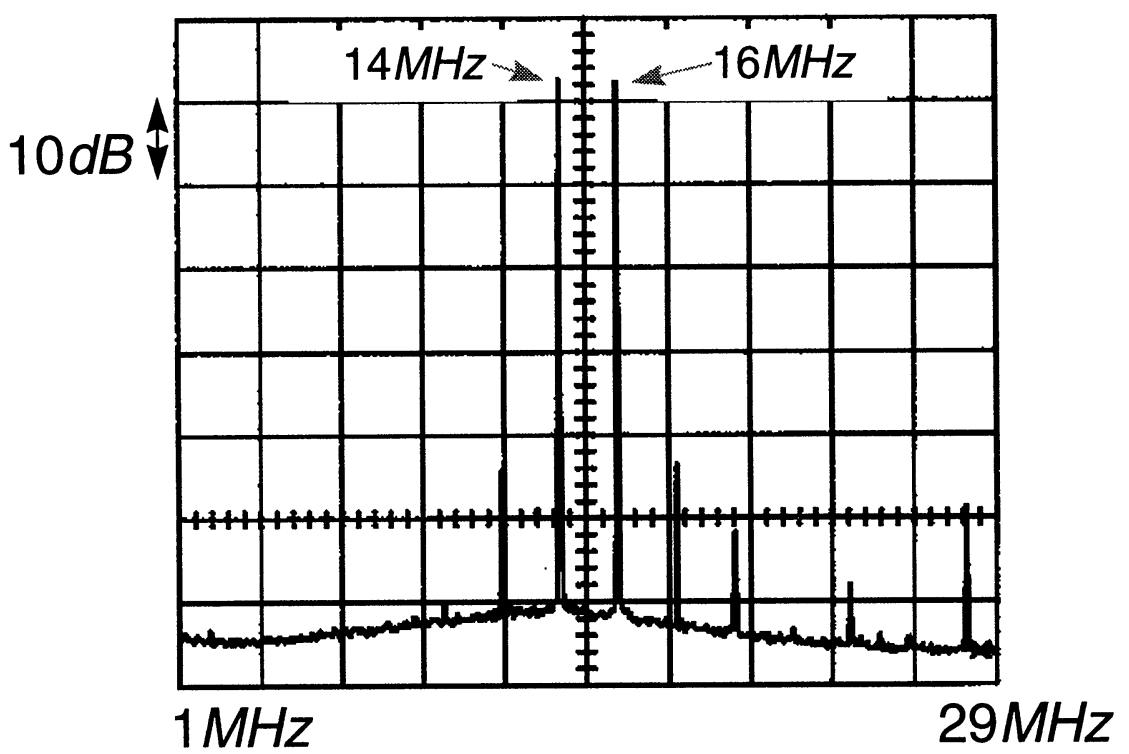

(b)

Figure 7 (a) Transient response when $D[2: 0]=000$, that is, cut-off frequency $=$ 15.0MHz. Top trace $=$ input, sum of $14.0 \mathrm{MHz}$ and $16.0 \mathrm{MHz}$ sinusoid. Bottom trace = filtered output. (b) Spectrum of the output in (a). Both of $14.0 \mathrm{MHz}$ and 16.0 $\mathrm{MHz}$ component can be seen. 


\section{CONCLUSION}

A low-voltage OTA is described and it is applied to a bandpass $\mathrm{Gm}-\mathrm{C}$ filter. The OTA can achieve high frequency operation with lowered power supply. A prototype sixth-order bandpass filter was implemented in $0.8 \mu \mathrm{m}$ CMOS process and the center frequency can be controlled from $15.0 \mathrm{MHz}$ to $33.0 \mathrm{MHz}$. The filter consumes $0.43 \mathrm{~mW}$ per pole with $3.0 \mathrm{~V}$ single power supply.

\section{REFERENCES}

A. Kaiser (1989) A micropower CMOS continuous-time low-pass filter. IEEE J. Solid-State Circuits, vol. SC-24, 736-743.

B. Nauta (1992) A CMOS transconductance-C filter technique for very high frequencies. IEEE J. Solid-State Circuits, vol. SC-27, no. 2, 142-153.

B. Stefanelli and A Kaiser (1993) A 2- $\mu \mathrm{m}$ CMOS fifth-order low-pass continuoustime filter for video frequency applications. IEEE J. Solid-State Circuits, vol. 28, no. 7, 713-718.

C. Yoo, S.-W. Lee, and W. Kim (1996) An automatic tuning scheme for Gm-C filters with an integrator as master. Dig. Tech. Papers, Symp. VLSI Circuits, 154-155.

F. Rezzi, A. Baschirotto, and R. Castello (1995) A 3V 12-55MHz BiCMOS pseudo-differential continuous-time filter. IEEE Trans. Circuits and Systems, Part-I, vol. 42, no. 11, 896-903.

J. M. Khoury (1991) Design of a 15-MHz CMOS continuous-time filter with onchip tuning. IEEE J. Solid-State Circuits, vol. SC-26, no. 12, 1988-1997.

H. Khorramabadi, M. J. Tarsia, and N. W. Woo (1996) Baseband filters for IS-95 CDMA receiver applications featuring digital automatic frequency tuning. Dig. Tech. Papers, International Solid-State Circuits Conference, 172-173.

P. K. D. Pai and A. A. Abidi (1994) A 40-mW 55-Mb/s CMOS equalizer for use in magnetic storage read channels. IEEE J. Solid-State Circuits, vol. 29, no. 4, 489-499.

Q. Huang (1995) A MOSFET-only continuous-time $560 \mathrm{kHz}$ tunable bandpass filter. Dig. Tech. Papers, Symp. VLSI Circuits, 93-94.

V. Gopinathan, Y. P. Tsividis, K.-S. Tan, and R. K. Hester (1990) Design considerations for high-frequency continuous-time filters and implementation of an antialiasing filter for digital video. IEEE J. Solid-State Circuits, vol. SC25 , no. $6,1368-1378$. 


\section{BIOGRAPHY}

Changsik Yoo was born in Daejon, Korea, on December 15, 1969. He received the B. S. degree summa cum laude, and M. S. degree in electronics engineering from Seoul National University, Seoul, Korea in 1992 and 1994, respectively, Since 1994, he has been working toward the $\mathrm{Ph}$. D. degree in the same university. In 1996, he was awarded silver prize in LG IC design contest held by LG semicon, Seoul, Korea. His research interests include analog circuit design and high speed interface circuits.

Wonchan Kim was born in Seoul, Korea, on December 11, 1945. He received the B. S. degree in electronics engineering from Seoul National University, Seoul, Korea, in 1972. He received the Dip.-Ing. and Dr.-Ing. degrees in electrical engineering from the Technische Hochschule Aachen, Aachen, Germany, in 1976 and 1981, respectively. Since 1982, he has been with School of Electrical Engineering, Seoul National University, where he is currently a professor. His research interests include development of semiconductor devices and design of analog/digital circuits. 